\title{
The three-dimensional noncommutative nonlinear sigma model in superspace
}

\author{
H.O. Girotti ${ }^{a}$, M. Gomes ${ }^{b}$, A.Yu. Petrov ${ }^{\text {b,c }}$, V.O. Rivelles ${ }^{\text {b }}$, A.J. da Silva ${ }^{\text {b }}$ \\ ${ }^{a}$ Instituto de Física, Universidade Federal do Rio Grande do Sul, Caixa Postal 15051, 91501-970, Porto Alegre, RS, Brazil \\ ${ }^{\mathrm{b}}$ Instituto de Física, Universidade de São Paulo, Caixa Postal 66318, 05315-970, São Paulo, SP, Brazil \\ ${ }^{\mathrm{c}}$ Department of Theoretical Physics, Tomsk State Pedagogical University, Tomsk 634041, Russia
}

Received 31 August 2001; accepted 27 September 2001

Editor: M. Cvetič

\begin{abstract}
We study the superspace formulation of the noncommutative nonlinear supersymmetric $O(N)$ invariant sigma-model in $2+1$ dimensions. We prove that the model is renormalizable to all orders of $1 / N$ and explicitly verify that the model is asymptotically free. (C) 2001 Elsevier Science B.V. All rights reserved.
\end{abstract}

PACS: $11.30 . \mathrm{Pb} ; 11.10 . \mathrm{Kk} ; 11.10 . \mathrm{Lm}$

Noncommutative field theories present many unusual properties. As a consequence of the noncommutativity, high momentum modes do not decouple from the physics at large distances leading to the appearance of infrared poles even in theories without massless particles. Being nonintegrable, these infrared singularities destroy the usual perturbative expansions. This fact has motivated many studies on the renormalization properties of noncommutative field theories [113].

Based on previous experience with commutative field theories one may wonder if supersymmetry is able to solve this problem without jeopardizing unitarity. This has been proven to be correct for the Wess-
Zumino model and also, at least to the subleading order of $1 / N$, for the nonlinear sigma model $[14,15]$. In this context, the use of the superspace formalism [16] has shown to be a very powerful tool to investigate noncommutative supersymmetric theories [17, 18]. In the present work, using superfield techniques to accommodate the intricacies of the Moyal product, we will demonstrate that the noncommutative nonlinear sigma model is renormalizable to all orders of $1 / N$. Furthermore, the renormalization group equations are analyzed and we prove that the model is asymptotically free.

The action of the noncommutative $O(N)$ sigma model in three-dimensional spacetime is $[15,19]$

$$
\begin{aligned}
S=\int d^{3} x d^{2} \theta \frac{1}{2}[ & \Phi_{j} D^{2} \Phi_{j} \\
& \left.\quad-\Sigma \star\left(\Phi_{j} \star \Phi_{j}-\frac{N}{g}\right)\right],
\end{aligned}
$$


where $\Phi_{i}, i=1, \ldots, N$, are real superfields and $\Sigma$ is a Lagrange multiplier superfield which enforces the constraint $\Phi_{j} \Phi_{j}=N / g$. Just for reference and to make contact with our previous work [15], we quote the field component expansions for $\Phi_{j}$ and $\Sigma$ :

$\Phi_{j}=\varphi_{j}+\bar{\theta} \psi_{j}+\frac{1}{2} \bar{\theta} \theta F_{j}$,

$\Sigma=\sigma+\bar{\theta} \xi+\frac{1}{2} \bar{\theta} \theta \lambda$,

where $\psi$ is a $N$ component Majorana spinor and $\varphi$ and $F$ are $N$ components scalar fields.

We are interested in a massive phase where $\Sigma$ acquires a nonvanishing vacuum expectation value. Thus, replacing $\Sigma \rightarrow \Sigma+m$, where $m$ is the vacuum expectation value of the original $\Sigma$, we obtain the new action

$$
\begin{aligned}
S=\int d^{3} x d^{2} \theta \frac{1}{2}[ & \Phi_{j}\left(D^{2}-m\right) \Phi_{j} \\
& \left.-\Sigma \star\left(\Phi_{j} \star \Phi_{j}-\frac{N}{g}\right)\right] .
\end{aligned}
$$

Using this expression it is straightforward to verify that the propagator for the basic superfields is

$$
\begin{aligned}
& \left\langle\widetilde{\Phi}_{i}\left(p_{1}, \theta_{1}\right) \widetilde{\Phi}_{j}\left(p_{2}, \theta_{2}\right)\right\rangle \\
& =i \frac{D^{2}+m}{p_{1}^{2}-m^{2}} \delta_{i j} \delta^{3}\left(p_{1}-p_{2}\right) \bar{\delta}_{12},
\end{aligned}
$$

where we have introduced the notation $\bar{\delta}_{12}=\delta\left(\bar{\theta}_{1}-\right.$ $\left.\bar{\theta}_{2}\right) \delta\left(\theta_{1}-\theta_{2}\right), D^{2}=\frac{1}{2} \bar{D} D$, the supercovariant derivative $D$ is

$D=\frac{\partial}{\partial \theta}-i \bar{\theta} \not \partial$

and $\bar{D}_{\alpha}=\gamma_{\alpha \beta}^{0} D_{\beta}$. The interaction vertex is given by

$$
\begin{aligned}
& \int d^{5} z \Sigma \star \Phi_{j} \star \Phi_{j} \\
& =\int d^{2} \theta \int \frac{d^{3} k_{1} d^{3} k_{2} d^{3} k_{3}}{(2 \pi)^{9}} \exp \left(-i \sum_{a<b} k_{a} \wedge k_{b}\right) \\
& \quad \times \widetilde{\Sigma}\left(k_{3}, \theta\right) \widetilde{\Phi}_{j}\left(k_{1}, \theta\right) \widetilde{\Phi}_{j}\left(k_{2}, \theta\right) \\
& \quad \times(2 \pi)^{3} \delta\left(k_{1}+k_{2}+k_{3}\right) \\
& \equiv \int d^{2} \theta \int \frac{d^{3} k_{1} d^{3} k_{2}}{(2 \pi)^{6}} \cos \left(k_{1} \wedge k_{2}\right) \\
& \quad \times \widetilde{\Sigma}\left(-k_{1}-k_{2}, \theta\right) \widetilde{\Phi}_{i}\left(k_{1}, \theta\right) \widetilde{\Phi}_{i}\left(k_{2}, \theta\right) .
\end{aligned}
$$

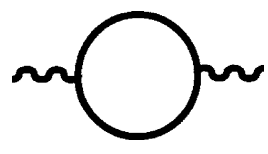

Fig. 1. Leading contribution to the two point proper function of the $\Sigma$ field. Continuous and wavy lines represent the $\Phi$ propagator and the external $\Sigma$ field.

Here, $k_{a} \wedge k_{b}=\frac{1}{2} k_{a \mu} \Theta^{\mu \nu} k_{b v}, \Theta_{\mu \nu}$ is the noncommutativity matrix and $\widetilde{\Phi}_{i}$ and $\widetilde{\Sigma}$ denote the Fourier transforms of the superfields $\Phi_{i}$ an $\Sigma$, respectively.

To find the effective propagator for the auxiliary field, let us consider the supergraph shown in Fig. 1. It is straightforward to verify that it contributes

$N \int d^{2} \theta \widetilde{\Sigma}(k, \theta)\left(D^{2}+2 m\right) \widetilde{\Sigma}(-k, \theta) I^{-1}\left(k^{2}\right)$,

where

$I^{-1}\left(k^{2}\right)=\frac{1}{2} \int \frac{d^{3} l}{(2 \pi)^{3}} \frac{\cos ^{2}(k \wedge l)}{\left(l^{2}-m^{2}\right)\left((k+l)^{2}-m^{2}\right)}$.

Using the relation [20]

$$
\begin{aligned}
& \int \frac{d^{n} k}{(2 \pi)^{n}} \frac{e^{i k \wedge p}}{\left(k^{2}+M^{2}\right)^{\lambda}} \\
& =\frac{M^{n / 2-\lambda}}{2^{\lambda-1}(2 \pi)^{n / 2} \Gamma[\lambda]} \frac{K_{n / 2-\lambda}\left(\sqrt{-M^{2} p \circ p}\right)}{(\sqrt{-p \circ p})^{n / 2-\lambda}},
\end{aligned}
$$

where $p \circ p=p_{\mu}\left(\Theta^{2}\right)^{\mu v} p_{\nu}$, we get

$$
\begin{aligned}
I^{-1}\left(k^{2}\right)= & \frac{i}{32 \pi} \int_{0}^{1} \frac{d x}{\sqrt{m^{2}-k^{2} x(1-x)}} \\
& \times\left[1-i \sqrt[4]{4 k \circ k\left(m^{2}-k^{2} x(1-x)\right)}\right. \\
& \times \sqrt{\frac{2}{\pi}} K_{-1 / 2} \\
& \left.\times\left(\sqrt{4 k \circ k\left(m^{2}-k^{2} x(1-x)\right)}\right)\right] .
\end{aligned}
$$

For large $k$ the last term either exponentially decreases or strongly oscillates; in both cases, its presence in a Feynman integral will lead to a finite result. For practical purposes, the dominant asymptotic behavior 
can be then taken as

$$
\begin{aligned}
I^{-1}\left(k^{2}\right) & \simeq \frac{i}{32 \pi} \int_{0}^{1} \frac{d x}{\sqrt{m^{2}-k^{2} x(1-x)}} \\
& \simeq \frac{i}{32 \sqrt{-k^{2}}} .
\end{aligned}
$$

In the sequel we are going to discuss the ultraviolet behavior of the $1 / N$ expansion for the model. To that end we find convenient to replace $I^{-1}\left(k^{2}\right)$ by the expression

$$
I^{-1}\left(k^{2}\right) \simeq \frac{i}{32 \sqrt{-k^{2}+4 m^{2}}},
$$

which has the same asymptotic behavior at $k \rightarrow \infty$ as (12). Of course, such replacement does not alter the leading UV behavior of Feynman integrals. Proceeding in this way, we obtain

$$
\begin{aligned}
& \left\langle\widetilde{\Sigma}\left(k_{1}, \theta_{1}\right) \widetilde{\Sigma}\left(k_{2}, \theta_{2}\right)\right\rangle \\
& \quad=-\frac{1}{2 N} I\left(k_{1}^{2}\right) \frac{D^{2}-2 m}{k_{1}^{2}-4 m^{2}} \delta_{12}^{5} \\
& \simeq-\frac{16 i}{N} \frac{D^{2}-2 m}{\sqrt{-k_{1}^{2}+4 m^{2}}} \delta^{3}\left(k_{1}-k_{2}\right) \bar{\delta}_{12} .
\end{aligned}
$$

The superficial degree of divergence associated to a generic Feynman supergraph $\gamma$ having, respectively, $n_{\Phi}$ and $n_{\Sigma}$ internal $\Phi$ and $\Sigma$ lines is given by

$d(\gamma)=3 L-2 n_{\Phi}-n_{\Sigma}+N_{D^{2}}$,

where $L$ is the number of loops and $N_{D^{2}}$ is the maximum number of $D^{2}$ factors which turn into loop momenta after the $\theta$ integration. Due to the properties of the $D^{2}$ derivatives [21], this number is

$N_{D^{2}}=n_{\Phi}+n_{\Sigma}-L=V-1$,

where $V$ is the number of vertices. Thus,

$d(\gamma)=2-N_{\Sigma}-\frac{N_{\Phi}}{2}$,

where $N_{\Sigma}$ and $N_{\Phi}$ are number of $\Sigma$ and $\Phi_{i}$ external lines, respectively. One should stress that (16) is actually an upper limit on the number of available $D^{2}$ factors since, after taking one $D^{2}$ factor for each loop, the number of those that may be converted into momentum must be even.

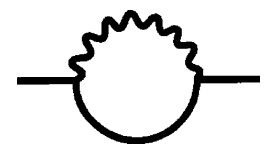

Fig. 2. $1 / N$ correction to the propagator of the $\Phi$ field.

Right away one sees that there is no quadratic divergences except for vacuum diagrams and, as we shall see shortly, there are neither linear divergences; hence this theory is free of the nonintegrable infrared divergences which spoil the usual perturbative expansions.

Before embarking into the general discussion, we shall analyze all possible cases of divergences at the leading order of $1 / N$. Linear divergences may arise in graphs having either $N_{\Sigma}=0, N_{\Phi}=2$ or $N_{\Sigma}=1$, $N_{\Phi}=0$. The last situation corresponds to the tadpole graph which, as we know, should be absent once the mass $m$ for the $\Phi$ field has been generated. Let us then consider the other possibility, which corresponds to the leading radiative correction to the $\Phi$ field propagator depicted in Fig. 2. By partially integrating, we may transfer the $D^{2}$ derivative from one of the propagators to the other lines. However, to get a nonvanishing result, only one factor of $D^{2}$ may survive in this process. Then, one of the $D^{2}$ has to be transferred to the external line and so the degree of divergence is lowered by one. At one-loop, just a logarithmic divergence may remain. Explicitly, the supergraph corresponds to

$$
\begin{aligned}
I_{1}= & \frac{16}{N} \int \frac{d^{3} p}{(2 \pi)^{3}} d^{2} \theta_{1} d^{2} \theta_{2} \widetilde{\Phi}_{i}\left(-p, \theta_{1}\right) \widetilde{\Phi}_{i}\left(p, \theta_{2}\right) \\
& \times \int \frac{d^{3} k}{(2 \pi)^{3}} \cos ^{2}(k \wedge p) \\
& \times \frac{\left(D^{2}-2 m\right) \bar{\delta}_{12}\left(D^{2}+m\right) \bar{\delta}_{12}}{\left((k+p)^{2}-m^{2}\right) \sqrt{-k^{2}+4 m^{2}}},
\end{aligned}
$$

which, after the aforementioned $D$-algebra transformation, can be cast as

$$
\begin{aligned}
I_{1}= & \frac{8}{N} \int \frac{d^{3} p}{(2 \pi)^{3}} d^{2} \theta \widetilde{\Phi}_{i}(-p, \theta)\left(D^{2}-m\right) \widetilde{\Phi}_{i}(p, \theta) \\
& \times\left[\int \frac{d^{3} k}{(2 \pi)^{3}} \frac{1}{\left(k^{2}-m^{2}\right) \sqrt{-k^{2}+4 m^{2}}}+\text { fin }\right],
\end{aligned}
$$




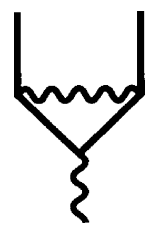

(a)

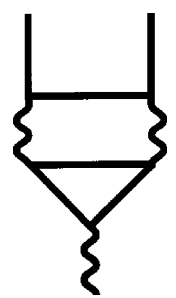

(b)
Fig. 3. Leading vertex corrections.

where fin indicates a finite contribution. In this Letter, all divergent integrals are to be understood as being dimensionally regularized. By integrating over $k$ and Fourier transforming with respect to the external momentum, we obtain

$$
I_{1}=-\frac{4 i}{\pi^{2} N} \int d^{5} z \Phi_{i}\left(D^{2}-m\right) \Phi_{i}\left(\frac{1}{\epsilon}+\text { fin }\right) .
$$

Notice that in the commutative case the divergence of the $\Phi$ propagator is the same as in (20). To finalize this preliminary discussion, we need to consider the leading corrections to the three point function. The relevant diagrams are shown in Fig. 3. The contributions of these supergraphs are, respectively,

$$
\begin{aligned}
I_{3 a}= & -\frac{16}{N} \int \frac{d^{3} p_{1} d^{3} p_{2}}{(2 \pi)^{6}} \int d^{2} \theta_{1} d^{2} \theta_{2} d^{2} \theta_{3} \\
& \times \widetilde{\Phi}_{i}\left(p_{1}, \theta_{1}\right) \widetilde{\Phi}_{i}\left(-p_{2}, \theta_{2}\right) \widetilde{\Sigma}\left(p_{2}-p_{1}, \theta_{3}\right) \\
& \times\left(D^{2}-2 m\right) \bar{\delta}_{12}\left(D^{2}+m\right) \bar{\delta}_{13}\left(D^{2}+m\right) \bar{\delta}_{32} \\
& \times \int \frac{d^{3} k}{(2 \pi)^{3}} \frac{\cos \left(k \wedge p_{1}\right) \cos \left(k \wedge p_{2}\right)}{\sqrt{-k^{2}+m^{2}}\left[\left(k+p_{1}\right)^{2}-m^{2}\right]} \\
& \times \frac{\cos \left(\left(k+p_{1}\right) \wedge\left(p_{1}-p_{2}\right)\right)}{\left[\left(k+p_{2}\right)^{2}-m^{2}\right]}
\end{aligned}
$$

and

$$
\begin{aligned}
I_{3 b}= & \frac{(16)^{2} i}{N} \int \frac{d^{3} p_{1} d^{3} p_{2}}{(2 \pi)^{6}} \\
& \times \int d^{2} \theta_{1} d^{2} \theta_{2} d^{2} \theta_{3} d^{2} \theta_{4} d^{2} \theta_{5} \\
& \times \widetilde{\Phi}_{i}\left(p_{1}, \theta_{1}\right) \widetilde{\Phi}_{i}\left(-p_{2}, \theta_{2}\right) \widetilde{\Sigma}\left(p_{2}-p_{1}, \theta_{5}\right) \\
& \times\left(D^{2}+m\right) \bar{\delta}_{12}\left(D^{2}-2 m\right) \bar{\delta}_{23}\left(D^{2}+m\right) \bar{\delta}_{34} \\
& \times\left(D^{2}-2 m\right) \bar{\delta}_{41}\left(D^{2}+m\right) \bar{\delta}_{45}\left(D^{2}+m\right) \bar{\delta}_{53}
\end{aligned}
$$

$$
\begin{aligned}
& \times \int \frac{d^{3} k d^{3} l}{(2 \pi)^{6}} \frac{\cos \left(k \wedge p_{1}\right) \cos \left(k \wedge p_{2}\right)}{\sqrt{-\left(k+p_{1}\right)^{2}+m^{2}}} \\
& \times \frac{\cos \left(\left(k+p_{1}\right) \wedge l\right) \cos \left(\left(k+p_{2}\right) \wedge l\right)}{\sqrt{-\left(k+p_{2}\right)^{2}+m^{2}}\left(k^{2}-m^{2}\right)\left(l^{2}-m^{2}\right)} \\
& \times \frac{\cos \left(\left(k-l+p_{1}\right) \wedge\left(p_{1}-p_{2}\right)\right)}{\left[\left(k-l+p_{1}\right)^{2}-m^{2}\right]\left[\left(k-l+p_{2}\right)^{2}-m^{2}\right]}
\end{aligned}
$$

Let us first consider $I_{3 a}$. It is straightforward to see that $\cos \left(k \wedge p_{1}\right) \cos \left(k \wedge p_{2}\right) \cos \left(\left(k+p_{1}\right) \wedge\left(p_{1}-p_{2}\right)\right)=$ $\frac{1}{4} \cos \left(p_{1} \wedge p_{2}\right)+\cdots$, where the dots indicate terms that depend on the internal momenta and thus will give finite contributions. Notice also that after integrating on $\theta_{3}$

$$
\begin{aligned}
& \left(D^{2}-2 m\right) \bar{\delta}_{12}\left(D^{2}+m\right) \bar{\delta}_{13}\left(D^{2}+m\right) \bar{\delta}_{32} \\
& \longrightarrow k^{2} \bar{\delta}_{12}+\cdots,
\end{aligned}
$$

due to the properties of the supercovariant derivatives. As a result of these manipulations, we can isolate the divergent part of $I_{3 a}$,

$$
\begin{aligned}
I_{3 a}= & -\frac{4}{N} \int \frac{d^{3} p_{1} d^{3} p_{2}}{(2 \pi)^{6}} \int d^{2} \theta \\
& \times \widetilde{\Phi}\left(p_{1}, \theta\right) \widetilde{\Phi}_{i}\left(-p_{2}, \theta\right) \widetilde{\Sigma}\left(p_{2}-p_{1}, \theta\right) \\
& \times \cos \left(p_{1} \wedge p_{2}\right) \int \frac{d^{3} k}{(2 \pi)^{3}} \frac{k^{2}}{\sqrt{-k^{2}+m^{2}}} \\
& \times \frac{1}{\left[\left(k+p_{1}\right)^{2}-m^{2}\right]\left[\left(k+p_{2}\right)^{2}-m^{2}\right]}+\cdots,
\end{aligned}
$$

so that the pole term is

$$
\begin{aligned}
I_{3 a}^{\mathrm{div}}= & \frac{2 i}{N \pi^{2} \epsilon} \int \frac{d^{3} p_{1} d^{3} p_{2}}{(2 \pi)^{6}} \int d^{2} \theta \widetilde{\Phi}_{i}\left(p_{1}, \theta\right) \\
& \times \widetilde{\Phi}_{i}\left(-p_{2}, \theta\right) \widetilde{\Sigma}\left(p_{2}-p_{1}, \theta\right) \cos \left(p_{1} \wedge p_{2}\right) .
\end{aligned}
$$

To obtain the corresponding divergence in the commutative case one should multiply this result by two and replace the cosine factor by one.

Concerning $I_{3 b}$, first notice that

$$
\begin{aligned}
\cos (k & \left.\wedge p_{1}\right) \cos \left(k \wedge p_{2}\right) \cos \left(\left(k+p_{1}\right) \wedge l\right) \\
\times & \cos \left(\left(k+p_{2}\right) \wedge l\right) \cos \left(\left(k-l+p_{1}\right) \wedge\left(p_{1}-p_{2}\right)\right) \\
\quad= & \frac{1}{16} \cos \left(p_{1} \wedge p_{2}\right)+\cdots .
\end{aligned}
$$


After performing the $\theta$ integrals, supercovariant derivative manipulations furnish now

$$
\begin{aligned}
& \left(D^{2}+m\right) \bar{\delta}_{12}\left(D^{2}-2 m\right) \bar{\delta}_{23}\left(D^{2}+m\right) \bar{\delta}_{34} \\
& \quad \times\left(D^{2}-2 m\right) \bar{\delta}_{41}\left(D^{2}+m\right) \bar{\delta}_{45}\left(D^{2}+m\right) \bar{\delta}_{53} \\
& \quad \longrightarrow\left(k+p_{2}\right)^{2}\left(k-l+p_{2}\right)^{2}+\cdots
\end{aligned}
$$

Using these results, one arrives at

$$
\begin{aligned}
I_{3 b}= & \frac{16 i}{N} \int \frac{d^{3} p_{1} d^{3} p_{2}}{(2 \pi)^{6}} \int d^{2} \theta \widetilde{\Phi}_{i}\left(p_{1}, \theta\right) \widetilde{\Phi}_{i}\left(-p_{2}, \theta\right) \\
& \times \widetilde{\Sigma}\left(p_{2}-p_{1}, \theta\right) \cos \left(p_{1} \wedge p_{2}\right) \\
& \times \int \frac{d^{3} k d^{3} l}{(2 \pi)^{6}} \frac{\left(k+p_{2}\right)^{2}\left(k-l+p_{2}\right)^{2}}{\sqrt{-\left(k+p_{1}\right)^{2}+m^{2}}} \\
& \times \frac{1}{\sqrt{-\left(k+p_{2}\right)^{2}+m^{2}}\left(k^{2}+m^{2}\right)\left(l^{2}-m^{2}\right)} \\
& \times \frac{1}{\left[\left(k-l+p_{1}\right)^{2}-m^{2}\right]\left[\left(k-l+p_{2}\right)^{2}-m^{2}\right]} \\
& + \text { fin, }
\end{aligned}
$$

which contains the following divergent part

$$
\begin{aligned}
I_{3 b}^{\mathrm{div}}= & \frac{i}{\pi^{2} N \epsilon} \int \frac{d^{3} p_{1} d^{3} p_{2}}{(2 \pi)^{6}} \int d^{2} \theta \widetilde{\Phi}_{i}\left(p_{1}, \theta\right) \\
& \times \widetilde{\Phi}_{i}\left(-p_{2}, \theta\right) \widetilde{\Sigma}\left(p_{2}-p_{1}, \theta\right) \cos \left(p_{1} \wedge p_{2}\right) .
\end{aligned}
$$

Summarizing, the divergent part of the vertex correction is

$$
S_{\Sigma}=\frac{3 i}{N \pi^{2} \epsilon} \int d^{5} z \Sigma \star \Phi_{i} \star \Phi_{i} .
$$

In the commutative case the corresponding result is

$$
\frac{8 i}{N \pi^{2} \epsilon} \int d^{5} z \Sigma \Phi_{i} \Phi_{i},
$$

so that, in view of (20), it may be eliminated by just a wave function renormalization of the $\Phi$ field. Unlike the commutative case, however, the renormalization of the vertex requires here also a wave function renormalization for the auxiliary field $\Sigma$. From a formal viewpoint, this is caused by the presence of additional factors $1 / 2^{V-1}$ in the planar contributions. These modifications are, of course, a consequence of the specific nonlocality induced by the Moyal products.

To complete the discussion of the renormalization at leading order we should examine the possible divergences associated with the four point function of the $\Phi$ field. By power counting, the four point function may be logarithmically divergent but this divergence is canceled. To see how this cancellation happens, consider the highest degree contribution in the internal (loop) momentum. It contains four $D^{2}$ factors but only two of them can be converted into momentum since one of those remaining must be associated to the loop integration to produce a nonvanishing result. Therefore, one of the $D^{2}$ factors does not contribute to the degree of divergence which becomes less by one than it was initially thought. Hence, the resulting contribution is finite.

From a formal standpoint, the divergences we found may be absorbed into field and coupling constant redefinitions

$$
\begin{aligned}
& \Phi_{i 0}=Z_{\Phi}^{1 / 2} \Phi_{i}, \\
& \Sigma_{0}=Z_{\Sigma} \Sigma, \\
& g_{0}=Z_{g} g,
\end{aligned}
$$

where $\Phi_{i 0}, \Sigma_{0}, g_{0}$ are the bare quantities and $\Phi_{i}, \Sigma, g$ the renormalized ones. From (20) we obtain

$Z_{\Phi}=1+\frac{8}{\pi^{2} N \epsilon}$,

which, as mentioned earlier, is the same as in the commutative case. Nevertheless, unlike the commutative case, the $Z_{\Sigma}$ renormalization constant is not trivial. In fact, from (30) we have

$Z_{\Sigma} Z_{\Phi}=1+\frac{6}{\pi^{2} N \epsilon}$,

from which

$Z_{\Sigma}=1-\frac{2}{N \pi^{2} \epsilon}$.

$Z_{g}$ is fixed by the mass gap equation. As this equation is not modified by the effect of the noncommutativity, an identical mass formula is to be expected. Indeed, the condition that $\Sigma$ has zero vacuum expectation value leads to

$\frac{i}{Z_{g} g}+Z_{\Phi} \int \frac{d^{3} k}{(2 \pi)^{3}} \frac{1}{k^{2}-m^{2}}=0$,

which after integration becomes

$\frac{1}{g}=-Z_{\Phi} Z_{g} \frac{m}{4 \pi}$, 
fixing $Z_{\Phi} Z_{g}$ to be a finite constant. By making the choice

$Z_{\Phi} Z_{g}=1-\frac{\mu}{m}$,

we obtain

$\frac{1}{g}=\frac{\mu}{4 \pi}-\frac{m}{4 \pi}$,

in agreement with the commutative case $[15,19]$. From this, it also follows that the theory is asymptotically free, the renormalization group beta function being given by

$\beta(g)=-\frac{\mu}{4 \pi} g$.

We are now in a position to prove that the model is renormalizable at any finite order of $1 / N$. The first observation is that supergraphs with two external $\Sigma$ lines have negative degree of divergence. Indeed, the number of vertices $V$ in such graphs is always even, $V-1$ is odd and, then, the number of $D^{2}$ factors that may be turned into momenta decreases by one from the value specified in (16). Hence, these supergraphs are superficially convergent. By the same reason, all supergraphs with two external $\Phi$ lines are at most logarithmically divergent. Indeed, the number of vertices is also even leading to the conclusion that one of the $D^{2}$ factors is superfluous and cannot be converted into momentum. Thus, the superficial degree of divergence decreases from one to zero. Analogous reasoning applied to the four point function of the $\Phi$ field shows that there is no overall divergence associated to the ultraviolet behavior of the graph as whole. We may conclude that there are at most logarithmic divergences and, therefore, only a mild integrable infrared singularity will appear. This is a power counting renormalization condition, which is necessary but not sufficient to guarantee that the model is renormalizable. It still remains to prove that the needed counterterms have the same Moyal product structure of those vertices already present in the original action. Specifically, one needs to show that, at any given order of $1 / N$, the divergent parts of the supergraphs with two $\Phi$ and one $\Sigma$ external lines generate a counterterm of the form $\int d^{5} z \Sigma \star \Phi_{i} \star \Phi_{i}$. This result follows from the statements:

1. Loop integrations associated to nonplanar subgraphs are finite. Indeed, any such (sub)graph contains a phase factor of the form [22]

$\Gamma=V(k, p) \exp \left(\frac{i}{2} \sum_{i, j} I_{i j} k_{i} \wedge k_{j}\right)$,

where $k$ and $p$ denote the sets of internal and external momenta, $I_{i j}$ is the intersection matrix whose entries are \pm 1 depending on whether the lines carrying momenta $k_{i}$ and $k_{j}$ cross from different sides and 0 otherwise. Thus, the analytic expression associated to such nonplanar subgraph must contain a factor of the form

$\int \frac{d^{3} k_{i}}{(2 \pi)^{3}} \frac{1}{\left(k_{i}^{2}-m^{2}\right)^{\alpha}} e^{i k_{i} \wedge l}(\cdots)$,

where the dots indicate terms which do not depend on $k_{i}, l$ is some linear combination of the external and internal momenta, and $\alpha$ is either an integer or half integer number. From (10) we see that the $k_{i}$ integral is finite and furthermore that the $l$ integration will contain a convergence factor as well. This shows that nonplanar subgraphs are superficially convergent. An immediate consequence of this fact is that the degree of divergence of an arbitrary graph is determined only by its planar subgraphs.

2. All divergent contributions associated to graphs with $N_{\Sigma}=1$ and $N_{\Phi}=2$ have the Moyal product structure $\int \Sigma \star \Phi \star \Phi$. This result follows from the property

$$
\begin{aligned}
& \cos a_{1} \cos a_{2} \cdots \cos a_{n} \\
& \quad=\frac{1}{2^{n-1}} \sum \cos \left(a_{1} \pm a_{2} \cdots \pm a_{n}\right),
\end{aligned}
$$

where the sum is taken over all possible combinations of the \pm signs. The above expression allows one to demonstrate that for any graph with an arbitrary number of loops there is one planar contribution, i.e., containing a cosine factor depending only on the external momenta. In fact, this result holds for an arbitrary (having any number of external lines) oneloop graph. To see that, consider the one-loop graph depicted in Fig. 4. From (43) we extract the following term

$$
\begin{aligned}
& \cos \left[k \wedge p_{1}+\left(k+p_{1}\right) \wedge p_{2}+\cdots\right. \\
& \left.\quad+\left(k+p_{1}+\cdots+p_{n-1}\right) \wedge p_{n}\right],
\end{aligned}
$$

which, after taking into account external momentum conservation, turns out not to depend on the loop 


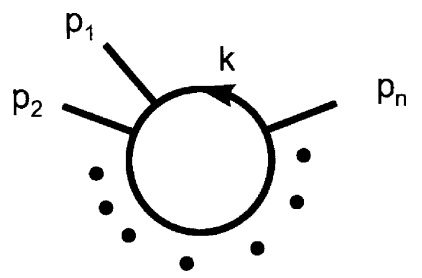

Fig. 4. One-loop contribution to the Green function.

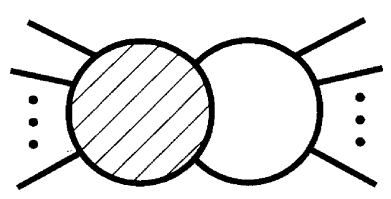

(a)

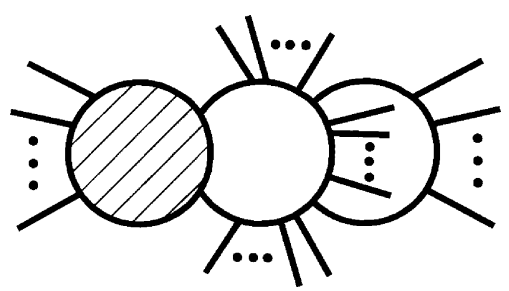

(b)

Fig. 5. (a) An $n$-loop contribution. The dashed circle stands for an arbitrary graph. (b) The $(n+1)$-loop graph obtained by joining two external lines through a tree structure.

momentum, as stated. We now assume that the result holds for an arbitrary $n$-loop graph. We may then increase the number of loops by one unity by joining two external lines through a tree diagram consisting of one line with possibly other external lines attached to it (see Fig. 5). Using the same procedure as in the oneloop case, we can verify again that there is one term whose cosine factor does not depend on the new loop momenta. This proves our statement.

As a concluding remark, we want to emphasize that, in spite of the nonlocality of the Moyal interaction, the noncommutative nonlinear sigma model remains renormalizable to all orders of $1 / N$, as we proved. The renormalization program is nevertheless modified as compared with the commutative case since a renormalization for the $\Sigma$ field is now required. Furthermore, the leading order correction, which is of or- der $N$, does not depend on the noncommutativity parameter. This dependence occurs in the next to the leading order and it is of the form $1 / \sqrt{\Theta^{2}}$, hence it does not possess a commutative limit.

\section{Acknowledgements}

This work was partially supported by Fundação de Amparo à Pesquisa do Estado de São Paulo (FAPESP) and Conselho Nacional de Desenvolvimento Científico e Tecnológico (CNPq). Two of us (H.O.G. and V.O.R.) also acknowledge support from PRONEX under contract CNPq 66.2002/1998-99. A.Yu.P. has been supported by FAPESP, project No. 00/12671-7.

\section{References}

[1] S. Minwalla, M.V. Raamsdonk, N. Seiberg, JHEP 0002 (2000) 020, hep-th/9912072;

M.V. Raamsdonk, N. Seiberg, JHEP 0003 (2000) 035, hepth/0002186.

[2] A. Matusis, L. Susskind, N. Toumbas, JHEP 0012 (2000) 002, hep-th/0002075.

[3] M. Hayakawa, Phys. Lett. B 478 (2000) 394, hep-th/9912094; M. Hayakawa, hep-th/9912167.

[4] I.Ya. Aref'eva, D.M. Belov, A.S. Koshelev, O.A. Rytchkov, Phys. Lett. B 487 (2000) 357.

[5] I.Ya. Aref'eva, D.M. Belov, A.S. Koshelev, Nucl. Phys. Suppl. 102 (2001) 17, hep-th/0003176.

[6] C.P. Martin, D. Sanchez-Ruiz, Phys. Rev. Lett. 83 (1999) 476, hep-th/9903077.

[7] H. Grosse, T. Krajewski, R. Wulkenhaar, hep-th/0001182.

[8] A. Armoni, hep-th/0005208.

[9] L. Bonora, M. Schnabl, A. Tomasiello, Phys. Lett. B 485 (2000) 311, hep-th/0002210.

[10] M.M. Sheikh-Jabbari, JHEP 9906 (1999) 015, hepth/9903107.

[11] I. Chepelev, R. Roiban, JHEP 0005 (2000) 037, hepth/9911098;

I. Chepelev, R. Roiban, JHEP 0103 (2001) 001.

[12] J.M. Gracia-Bondia, C.P. Martin, Phys. Lett. B 479 (2000) 321, hep-th/0002171.

[13] For a review, see M.R. Douglas, N.A. Nekrasov, hepth/0106048, to appear in Rev. Mod. Phys.

[14] H.O. Girotti, M. Gomes, V.O. Rivelles, A.J. da Silva, Nucl. Phys. B 587 (2000) 299, hep-th/0007080.

[15] H.O. Girotti, M. Gomes, V.O. Rivelles, A.J. da Silva, hepth/0102101.

[16] A. Bichl, J.M. Grimstrup, H. Grosse, L. Popp, M. Schweda, R. Wulkenhaar, JHEP 0010 (2000) 046, hep-th/0007050.

[17] D. Zanon, Phys. Lett. B 502 (2001) 265; D. Zanon, Phys. Lett. B 504 (2001) 101. 
[18] I.L. Buchbinder, M. Gomes, A.Yu. Petrov, V.O. Rivelles, hepth/0107022, to appear in Phys. Lett. B.

[19] V.G. Koures, K.T. Mahantappa, Phys. Rev. D 43 (1991) 3428; V.G. Koures, K.T. Mahantappa, Phys. Rev. D 45 (1992) 480.

[20] I.M. Gel'fand, G.E. Shilov, Generalized Functions, Vol. 1, Academic Press, 1964.
[21] S.J. Gates Jr., M.T. Grisaru, M. Rocek, W. Siegel, Superspace or One Thousand and One Lessons in Supersymmetry, Benjamin/Cummings, 1983.

[22] T. Filk, Phys. Lett. B 376 (1996) 53. 